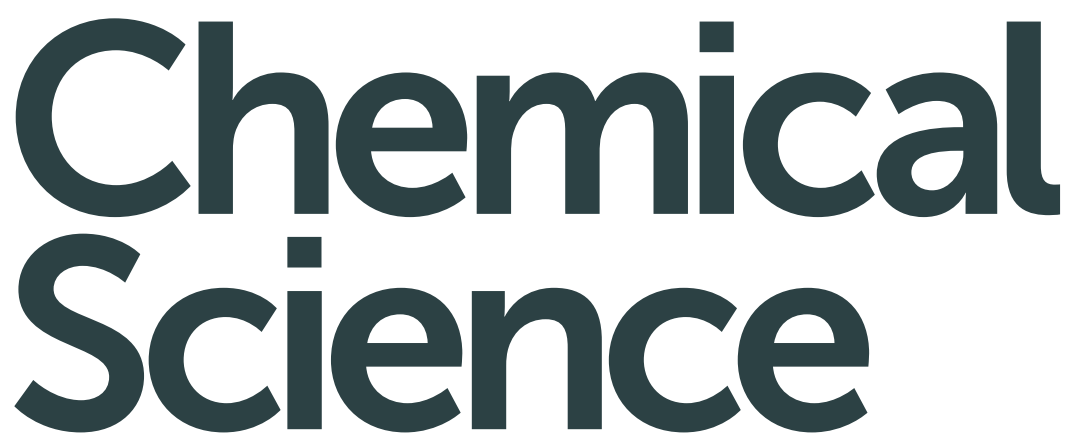

rsc.li/chemical-science
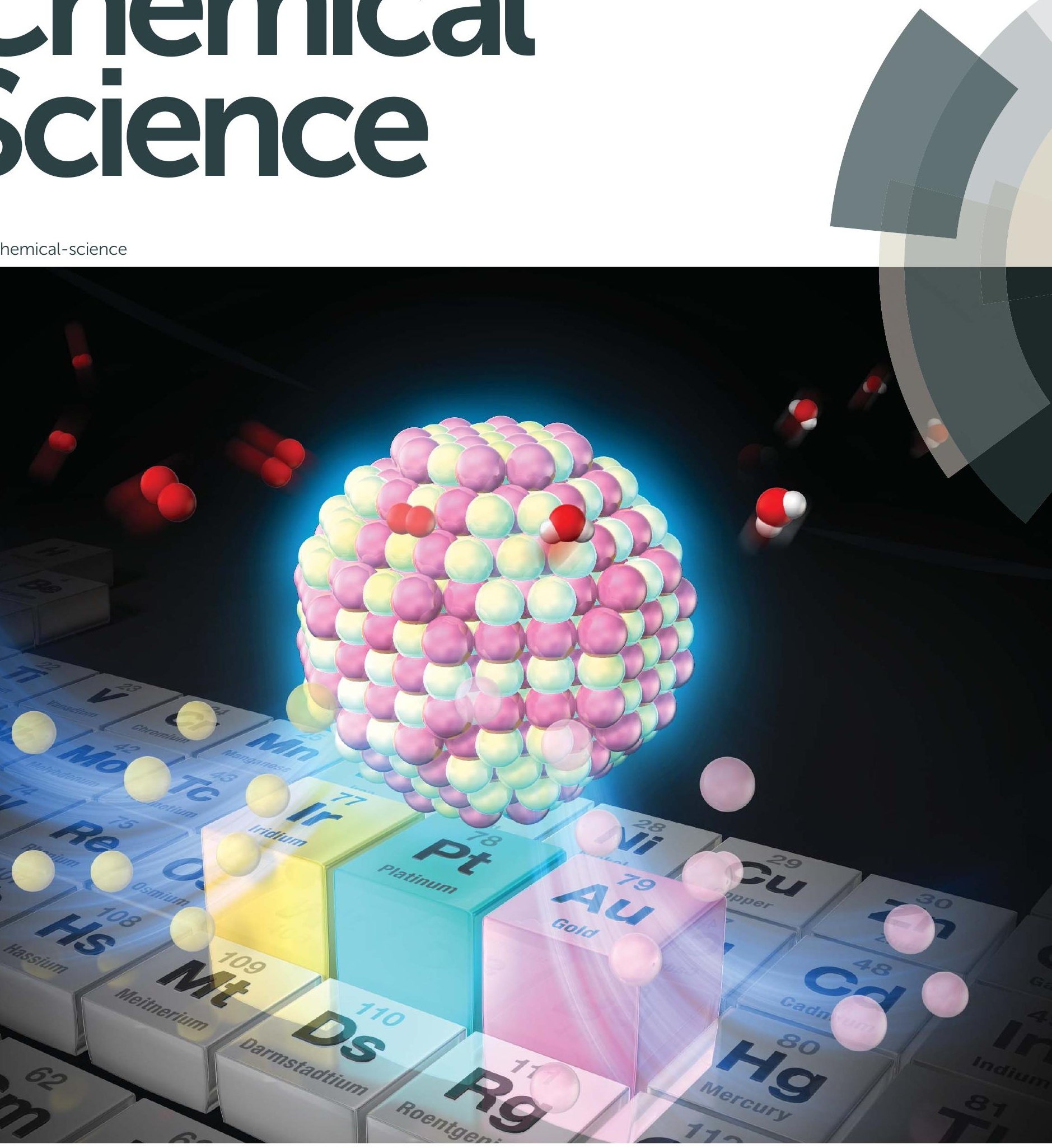

ISSN 2041-6539

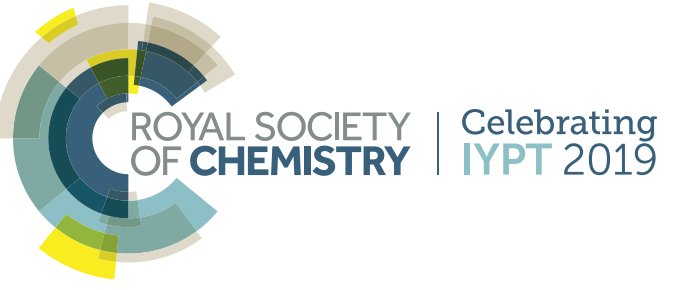

EDGE ARTICLE

Kohei Kusada, Michihisa Koyama, Hiroshi Kitagawa et al. Emergence of high ORR activity through controlling local density-of-states by alloying immiscible Au and Ir 


\section{Check for updates}

Cite this: Chem. Sci., 2019, 10, 652

๑ All publication charges for this article have been paid for by the Royal Society of Chemistry

Received 17th September 2018 Accepted 26th November 2018

DOI: $10.1039 / \mathrm{c} 8 \mathrm{sc} 04135 \mathrm{k}$

rsc.li/chemical-science

\title{
Emergence of high ORR activity through controlling local density-of-states by alloying immiscible Au and Ir
}

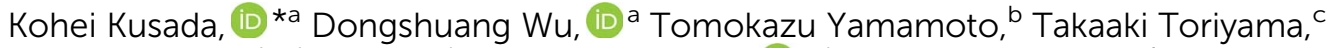 \\ Syo Matsumura, ${ }^{\text {bcd }}$ Wei Xie, ${ }^{d}$ Michihisa Koyama, (D) *de Shogo Kawaguchi, ${ }^{f}$ \\ Yoshiki Kubota ${ }^{9}$ and Hiroshi Kitagawa (D) *ad
}

\begin{abstract}
The electronic structure of surface atoms has a great effect on catalytic activity because the binding energy of reactants is closely related to the electronic structure. Therefore, designing and controlling the local density of states (LDOS) of the catalyst surface would be a rational way to develop innovative catalysts. Herein, we first demonstrate a highly active Aulr solid-solution alloy electrocatalyst for the oxygen reduction reaction (ORR) by emulating the Pt LDOS profile. The calculated LDOS of Ir atoms on the $\mathrm{Au}_{0.5} \mathrm{Ir}_{0.5}(111)$ surface closely resembled that of $\mathrm{Pt}(111)$, resulting in suitable oxygen adsorption energy on the alloy surface for the ORR. We successfully synthesized Aulr solid-solution alloys, while Ir and Au are immiscible even above their melting points in the bulk state. Although monometallic Ir or Au is not active for the ORR, the synthesized $\mathrm{Au}_{0.5} \mathrm{Ir}_{0.5}$ alloy demonstrated comparable activity to Pt at $0.9 \mathrm{~V}$ versus a reversible hydrogen electrode.
\end{abstract}

\section{Introduction}

Designing the electronic structure of materials is one of the best ways to tune the material properties because not only physical properties such as magnetic and electrical properties but also chemical properties such as catalytic properties are closely related to the density of states (DOS) of solids. ${ }^{1,2}$ In particular, for catalytic reactions, the local DOS (LDOS), which is a projection of DOS to each atom, of the surface atoms is supposed to be more important than the total DOS of a bulk solid because reactants interact with atoms on a solid surface, and the catalytic activity is crucially affected by the binding energy of reactants on the catalyst surface. Therefore, LDOS engineering

${ }^{a}$ Division of Chemistry, Graduate School of Science, Kyoto University, Kitashirakawa Oiwake-cho, Sakyo-ku, Kyoto 606-8502, Japan. E-mail: kusada@kuchem.kyoto-u.ac. jp; kitagawa@kuchem.kyoto-u.ac.jp

${ }^{b}$ Department of Applied Quantum Physics and Nuclear Engineering, Kyushu University, 744 Motooka, Nishi-ku, Fukuoka 819-0395, Japan

'The Ultramicroscopy Research Center, Kyushu University, 744 Motooka, Nishi-ku, Fukuoka 819-0395, Japan

${ }^{d}$ INAMORI Frontier Research Center, Kyushu University, 744 Motooka, Nishi-ku, Fukuoka 819-3095, Japan

${ }^{e}$ GREEN, National Institute for Materials Science, 1-1 Namiki, Tsukuba, Ibaraki 3050044, Japan. E-mail: koyama.michihisa@nims.go.jp

fJapan Synchrotron Radiation Research Institute (JASRI) SPring-8, 1-1-1 Kouto, Sayocho, Sayo-gun, Hyogo 679-5198, Japan

${ }^{g}$ Department of Physical Science, Graduate School of Science, Osaka Prefecture University, 1-1 Gakuen-cho, Naka-ku, Sakai, Osaka 599-8531, Japan

$\dagger$ Electronic supplementary information (ESI) available. See DOI: $10.1039 / \mathrm{c} 8 \mathrm{sc} 04135 \mathrm{k}$ would realize an innovative catalyst possessing the desirable LDOS profile for targeted reactions. As a demonstration, we aimed to create pseudo-Pt catalysts by emulating the electronic structure of Pt with other elements.

A solid-solution alloy is a favourable material to continuously control its electronic structure. In such an alloy, the constituents randomly mix at the atomic level, and thus, we can continuously control its electronic structure by changing the combination of elements and/or composition of constituents. However, even among d-block elements, the majority of bulk alloy systems are the immiscible type under ambient conditions, hindering us from freely developing solid-solution alloys and designing DOS profiles. Recently, we demonstrated that the nanosize effect offers a chance to find a way out of this metallurgical difficulty; that is, we can obtain metal nanoparticles (NPs) having new phases that do not exist in bulk states. ${ }^{3-6}$ In general, a nanoparticle with the same crystal structure as the corresponding bulk material is obtained. However, if we find appropriate synthetic conditions, it allows us to develop novel NPs adopting new phases including solid-solution alloy NPs consisting of immiscible combinations. ${ }^{6-11}$ Therefore, by focusing on NPs, we would be able to synthesize any solidsolution alloys regardless of the bulk phase diagrams, leading us to create effective catalysts.

On the basis of LDOS engineering, we have focused on an AuIr solid-solution alloy as one of the candidates for pseudo-Pt because Pt is located between Ir and Au on the periodic table of the elements. However, these elements cannot mix with each other at the atomic level, even above their melting points. ${ }^{12}$ 
Although several papers have been reported on the $\mathrm{Au}-\mathrm{Ir}$ system, most of them were on the segregated type, and a pure solid-solution alloy had not been obtained. ${ }^{\mathbf{1 3 - 2 0}}$.

In this study, we emulate the Pt LDOS profile and create AuIr solid-solution alloy NPs over the whole metallic composition. We demonstrate that the atomic-level alloying can realize a Ptlike electronic structure at the Ir atoms on the $\mathrm{Au}_{0.5} \operatorname{Ir}_{0.5}(111)$ surface, and $\mathrm{Au}_{0.5} \mathrm{Ir}_{0.5}$ exhibits comparable oxygen reduction reaction (ORR) activity to that of Pt. To design the Pt-like LDOS and its catalytic properties, we calculated the electronic structure and oxygen binding energy of the alloy using density functional theory (DFT) method. The solid-solution alloy NPs of the bulk-immiscible $\mathrm{Au}$ and Ir combination were successfully synthesized by a chemical reduction method. The structure of the materials was characterized by atomic-resolution scanning transmission electron microscopy (STEM) coupled with energydispersive X-ray (EDX) spectroscopy and synchrotron X-ray diffraction (XRD). To confirm the emergence of Pt-like property, we examined the ORR activity because the ORR is wellknown as the reaction for which $\mathrm{Pt}$ shows excellent catalytic activity.

\section{Results and discussion}

\section{DFT calculations of the electronic structure and oxygen binding energies}

DFT calculations were performed to investigate the electronic structures of monometallic Ir, Pt and $\mathrm{Au}$, as well as $\mathrm{Au}_{x} \mathrm{Ir}_{1-x}$ alloys. Fig. 1a shows the comparison of the electronic structures of the (111) surface, which is the most stable surface of the facecantered cubic (fcc) structure. It is clearly seen that the electronic structure of $\mathrm{Au}_{x} \mathrm{Ir}_{1-x}$ is continuously changed from the Ir wider d-band to the $\mathrm{Au}$ narrower d-band, and $\mathrm{Au}_{0.5} \mathrm{Ir}_{0.5}$ possesses a unique electronic structure, which is totally different from monometallic Ir or $\mathrm{Au}$. The d-band width of $\mathrm{Au}_{0.5} \mathrm{Ir}_{0.5}$ is most similar to that of $\mathrm{Pt}$, which is shown as blue dotted lines. Pt shows several characteristic DOS peaks and the peaks located near the Fermi energy are relatively high.
Although the total DOS profile of the top layer of $A u_{0.5} \operatorname{Ir}_{0.5}$ is not similar to the Pt profile around the Fermi energy, the LDOS of Ir atoms of the top layer have high DOS near the Fermi energy. Hence, we focused on the LDOS of Ir and $\mathrm{Au}$ atoms on the $\mathrm{Au}_{0.5} \mathrm{Ir}_{0.5}$ alloy surface. Fig. $1 \mathrm{~b}$ and $\mathrm{S} 1$ in the ESI $\dagger$ show the LDOS of Ir or Au atoms on the alloy surface as well as the LDOS of Pt atoms on the Pt surface for comparison. It is notable that the LDOS of Ir atoms on the alloy surface is similar to the Pt LDOS profile, whereas $\mathrm{Au}$ atoms show a quite different profile. We further investigated the oxygen binding energies on those surfaces to validate if the alloy can exhibit Pt-like ORR activity because ORR is well-known as a reaction in which Pt shows excellent catalytic activity. It is well-known that the binding energy of $\mathrm{O}$ is considered to be a significant factor for the ORR activity, ${ }^{21}$ and the ORR activity shows a volcano type dependence on the oxygen binding energy because too weak or too strong of a binding energy cannot promote the ORR. ${ }^{21,22}$ Consequently, it is considered that a catalyst having a proper $\mathrm{O}$ binding energy can provide high ORR activity. The $\mathrm{O}$ binding energies on each surface are summarized in Tables S3-S8. $\dagger$ Compared to monometallic surfaces, there are a variety of adsorption sites on the alloy surface, and we calculated the $\mathrm{O}$ binding energies for all possible adsorption sites on the $\mathrm{Au}_{0.5} \mathrm{Ir}_{0.5}$ surface model (Table S6 and Fig. S5 $\dagger$ ). Since the DOS changes by the atomic orders, we adopted a random alloy surface model to obtain standard adsorption energies on the alloy surface. However, the result shown here is one of the typical examples. The calculated results demonstrated that the adsorption sites involve mainly Ir atoms, which provide binding energies similar to that of Pt. Even if an $\mathrm{O}$ atom approaches the sites consisting of only $\mathrm{Au}$ atoms, the $\mathrm{O}$ atom moves to another site including Ir atoms. Therefore, Ir is considered to play a key role in the $\mathrm{O}$ adsorption on the alloy surface. These results show that the Pt-like LDOS profile is realized by the formation of an $\mathrm{Au}_{0.5} \mathrm{Ir}_{0.5}$ solid-solution alloy causing the change in composition and lattice parameter of alloy surface, and this LDOS provides similar $\mathrm{O}$ adsorption energies to $\mathrm{Pt}$, suggesting that $\mathrm{Au}_{0.5} \mathrm{Ir}_{0.5}$ shows Pt-like ORR activity.
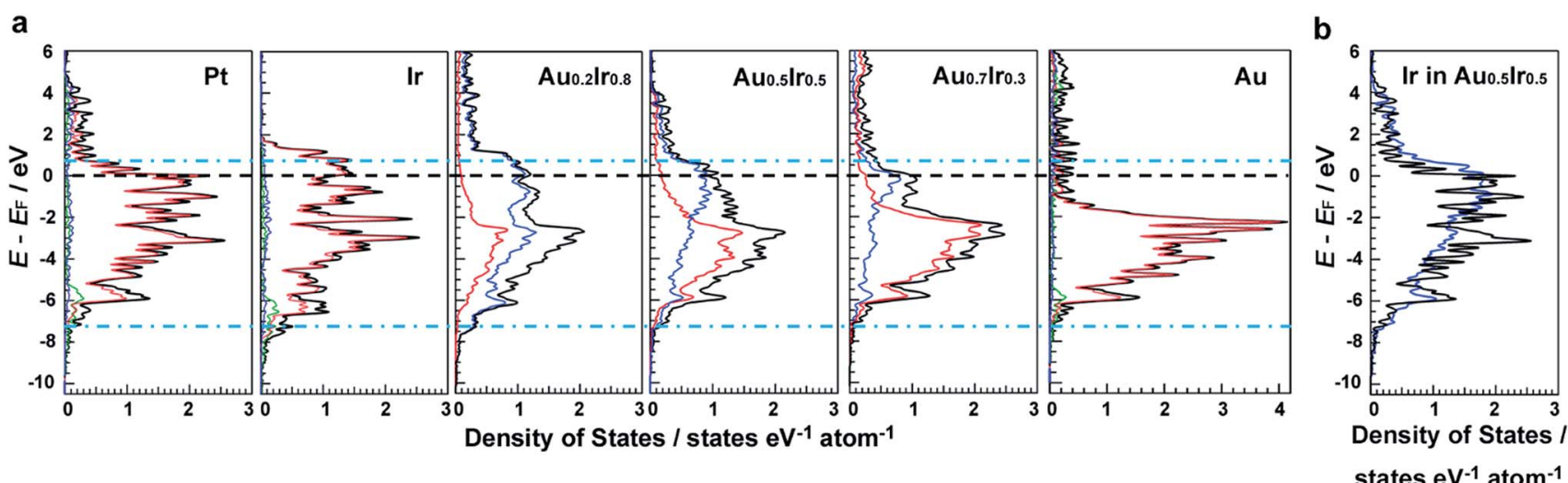

Fig. 1 The electronic structures of the surfaces of the catalysts studied by DFT calculations. (a) DOS of the top layer of Pt, Ir, Au and Au $\mid \mathrm{r}_{1-x}(111)$ surfaces. Blue and red lines in the $\mathrm{Au}_{x} \mathrm{I} \mathrm{r}_{1-x}$ solid-solution alloy indicate the $\mathrm{Ir}$ and $\mathrm{Au}$ LDOS, respectively. Red, green and blue lines in the Pt, Ir and Au surfaces show d-, s- and p-bands, respectively. (b) Comparison of the LDOS of the $A u_{0.5} I_{0.5}$ surface with the LDOS of the Pt surface (blue, IrLDOS in the $\mathrm{Au}_{0.5} \mathrm{Ir}_{0.5}$ solid-solution alloy; black, Pt). 


\section{Synthesis and characterization}

Due to the immiscible alloy system, synthesizing pure AuIr solid-solution alloy is still challenging even on the nanoscale. To overcome this challenge, we adopted a chemical reduction method that enables us to rapidly reduce different metal ions concurrently under extreme conditions. The method is very simple, as shown in Fig. 2. Both $\mathrm{HAuCl}_{4} \cdot 3 \mathrm{H}_{2} \mathrm{O}$ and $\operatorname{IrCl}_{4} \cdot \mathrm{H}_{2} \mathrm{O}$ were dissolved into deionized water. Meanwhile, poly( $N$-vinyl-2pyrrolidone) (PVP), a protecting agent, was added to ethylene glycol (EG), a reducing agent, and the solution was heated to $195{ }^{\circ} \mathrm{C}$. The precursor solution was then slowly added to the EG solution, and the metal ions were concurrently reduced in a moment. After the reduction finished, the prepared NPs were separated by centrifugation. On the other hand, if the precursors are dissolved in EG solution before heating, Au ions would be reduced earlier at lower temperature before Ir ions are reduced and then form phase separated NPs due to the difference of reduction potentials and the nature of immiscible $\mathrm{Au}-\mathrm{Ir}$ system.

The metal composition was controlled by the nominal ratio of the metal precursors, and three types of AuIr NPs were synthesized with different compositions. The metal composition and size of the obtained materials were characterized by $\mathrm{X}$ ray fluorescence (XRF) measurements and transmission electron microscopy (TEM), respectively (Tables S10 and S11 and Fig. S9†). We obtained $\mathrm{Au}_{0.2} \mathrm{Ir}_{0.8}(5.5 \pm 1.4 \mathrm{~nm}), \mathrm{Au}_{0.5} \mathrm{Ir}_{0.5}(7.2 \pm$ $1.7 \mathrm{~nm})$ and $\mathrm{Au}_{0.7} \mathrm{Ir}_{0.3}(9.9 \pm 2.3 \mathrm{~nm})$ NPs and found that the size of the NPs decreased with increasing Ir content under the same synthetic conditions.

Fig. 3a-c show a high-angle annular dark-field (HAADF) STEM image of $\mathrm{Au}_{0.5} \mathrm{Ir}_{0.5}$ NPs and the corresponding $\mathrm{Au}$ and $\mathrm{Ir}$ elemental maps. These results revealed that both Ir and $\mathrm{Au}$ atoms were distributed in all of the particles. Furthermore, an atomic resolution HAADF-STEM image is shown in Fig. 3d, and EDX line profiles of Ir-M and Au-M (Fig. 3e) were taken along the white arrow in Fig. 3d. From this result, we found that although the particle was polycrystalline, the two elements were existed in the same crystal grains; thus, the obtained NPs formed a solidsolution structure. The metal composition of the surface of $\mathrm{Au}_{0.5} \mathrm{Ir}_{0.5} \mathrm{NP}$ was further investigated by EDX point analysis (Fig. S10 $\dagger$ ). The average composition $x$ in $\mathrm{Au}_{x} \mathrm{Ir}_{1-x}$ was calculated to be $0.43 \pm 0.13$. Fig. $\mathrm{S} 11$ and $\mathrm{S} 12 \uparrow$ show that the obtained $\mathrm{Au}_{0.2} \mathrm{Ir}_{0.8}$ and $\mathrm{Au}_{0.7} \mathrm{Ir}_{0.3}$ also form solid-solution structures.

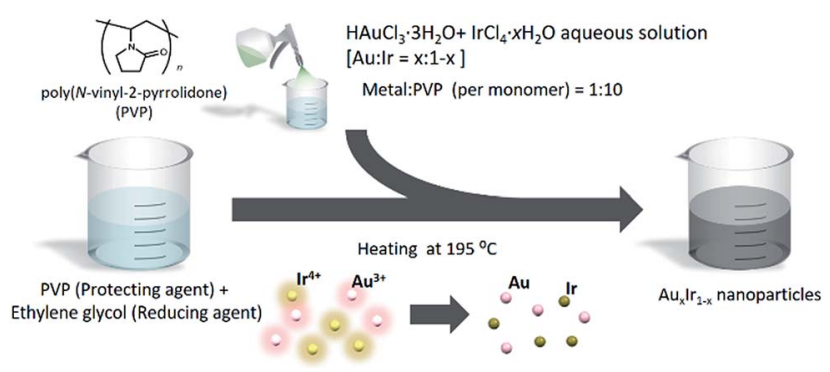

Fig. 2 Schematic image of the synthesis of $A u_{x} \mid r_{1-x}$ solid-solution alloy NPs.
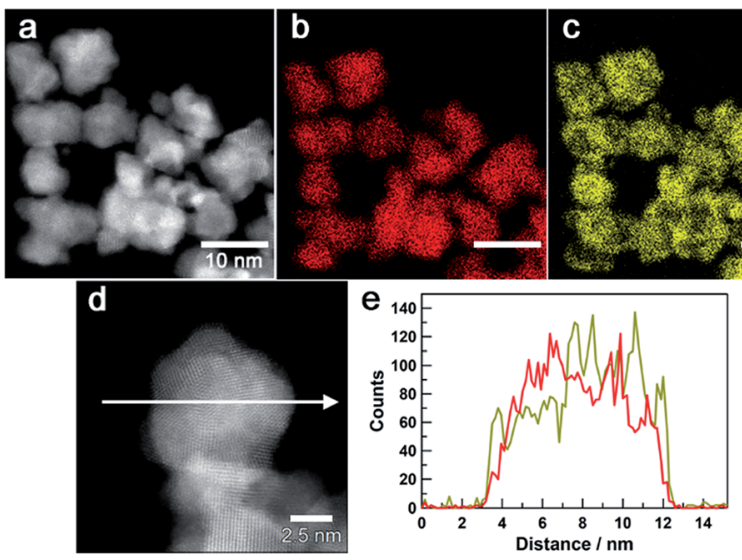

Fig. 3 Scanning transmission electron microscopy images of $A u_{0.5} \mid r_{0.5}$ NPs. (a) a HAADF-STEM image. (b), (c) Au-M and Ir-M STEM-EDX maps (red, Au; yellow, Ir). (e) Compositional line profiles of Au (red) and Ir (dark yellow) for the $\mathrm{Au}_{0.5} \mathrm{I} \mathrm{r}_{0.5}$ nanoparticle recorded along the arrow shown in (d) the HAADF-STEM image.

The crystal structures of the obtained $A u_{x} \operatorname{Ir}_{1-x}$ NPs were confirmed by synchrotron XRD analysis at the beamline BL02B2, SPring-8. ${ }^{23}$ Fig. 4a shows the XRD patterns of $A u_{x} \operatorname{Ir}_{1-x}$ NPs, the Ir and Au bulk powders. All of the $A u_{x} \mathrm{Ir}_{1-x}$ NPs, as well as the Ir and $\mathrm{Au}$ metals, show a single fcc pattern, and the diffraction peaks of $\mathrm{Au}_{x} \mathrm{Ir}_{1-x}$ NPs continuously shifted to lower angles with increasing $\mathrm{Au}$ content. Fig. $4 \mathrm{~b}$ shows the lattice constants of $\mathrm{Au}_{x} \mathrm{Ir}_{1-x}$ NPs, as determined by LeBail refinement of the XRD patterns. The lattice constants linearly increased with increasing Au content, and this linear correlation between the metal composition and the lattice constant follows Vegard's law, ${ }^{24}$ a well-known empirical rule for solid-solution materials. These results support that we first succeeded in synthesizing pure solid-solution AuIr alloy over the entire composition range.

\section{Catalytic properties}

To demonstrate the emergence of Pt-like property, we investigated the ORR activity of the AuIr alloy NPs as a probe reaction. We also synthesized monometallic Ir, Pt and Au NPs by chemical reduction methods to compare the ORR activity (see the
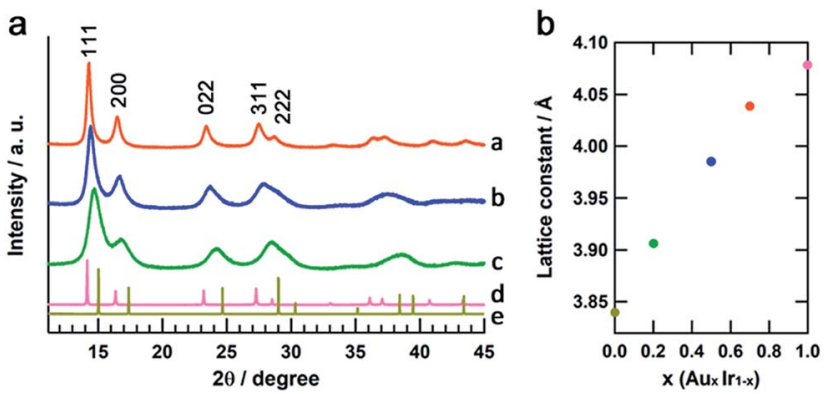

Fig. 4 X-ray diffraction analysis of $A u_{x} \mid r_{1-x}$ NPs. (a) Powder XRD patterns of $a ; A u_{0.7} \mid r_{0.3}$ NPs, b; $A u_{0.5} I_{0.5}$ NPs, c; $A u_{0.2} \mid r_{0.8}$ NPs, d; Au and e; Ir powders. (b) A plot of the changes in the lattice constants verses the metal composition. 
ESI $\dagger$ ). These monometallic NPs were prepared to have similar average diameters to that of $\mathrm{Au}_{0.5} \mathrm{Ir}_{0.5}$ to avoid the size effect, but we were not able to control the size of Ir NPs. All of the obtained NPs were supported on carbon black (Vulcan XC-72R), and the amount of metal in the catalysts was fixed at $20 \mathrm{wt} \%$. Measurements were performed with a rotating disk electrode (RDE) at a scanning speed of $5 \mathrm{mV} \mathrm{s}^{-1}$ in $1.0 \mathrm{M} \mathrm{NaOH}$ aqueous solution. The current density was normalized by the glassy carbon electrode geometric area $\left(0.196 \mathrm{~cm}^{2}\right)$. The polarization curves in Fig. 5a and b show that Pt catalyzes the reaction at a much lower overpotential compared to the Ir and Au catalysts, as is the case in previous reports..$^{25,26}$ It is notable that the $\mathrm{Au}_{0.5} \mathrm{Ir}_{0.5}$ alloy begins to catalyze the reaction at approximately $1.0 \mathrm{~V}$ versus the reversible hydrogen electrode (RHE), which is comparable to the onset potential of the Pt catalyst. Fig. 5c shows the comparison of the kinetic current density $\left(j_{\mathrm{k}}\right)$ on each catalyst measured at $0.9 \mathrm{~V}_{\mathrm{RHE}}$. The kinetic current was calculated based on the Koutecky-Levich equation as follows:

$$
1 / i=1 / i_{\mathrm{k}}+1 / i_{\mathrm{d}}
$$

where $i, i_{\mathrm{k}}$, and $i_{\mathrm{d}}$ are the measured current, kinetic current and diffusion-limiting current, respectively. $\mathrm{Au}_{0.5} \mathrm{Ir}_{0.5}$ shows a comparable kinetic current density to that of $\mathrm{Pt}$ at this potential. Furthermore, the Tafel slopes of the $\mathrm{Pt}, \mathrm{Au}_{0.5} \mathrm{Ir}_{0.5}, \mathrm{Au}$

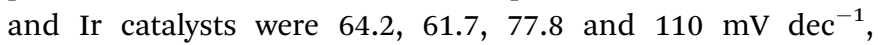
respectively (Fig. 5d). The slope of $\mathrm{Au}_{0.5} \mathrm{Ir}_{0.5}$ is smaller than those of $\mathrm{Au}$ and Ir; this slope suggests the improvement of the kinetics for the reaction. Interestingly, the similarity of the slopes of $\mathrm{Pt}$ and $\mathrm{Au}_{0.5} \mathrm{Ir}_{0.5}$ implies that the reaction mechanism
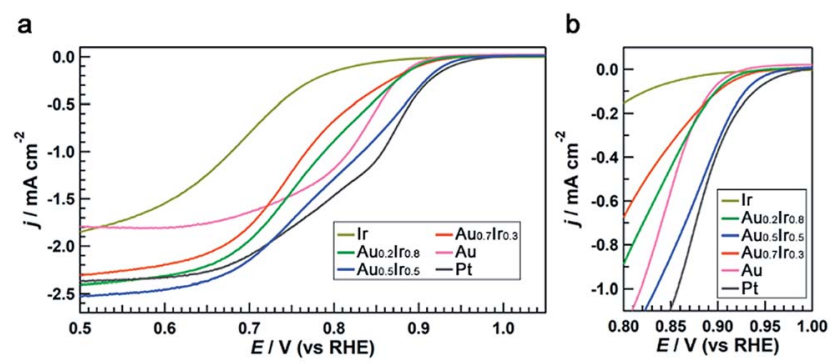

c

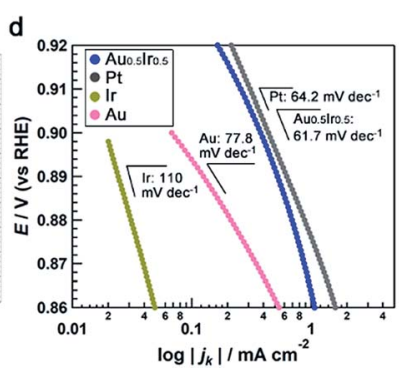

Fig. 5 Electrochemical oxygen-reduction activities of $A u_{x} \mid r_{1-x}$ alloy catalysts. (a), (b) rotating disk electrode $I-V$ polarization curves taken in $\mathrm{O}_{2}$-saturated $1.0 \mathrm{M} \mathrm{NaOH}$ aqueous solution normalized by the glassy carbon electrode geometric area $\left(0.196 \mathrm{~cm}^{2}\right)$ (a) from 0.50 to 1.05 $\mathrm{V}_{\mathrm{RHE}}$ and (b) from 0.80 to $1.00 \mathrm{~V}_{\mathrm{RHE}}$. (c) Summary of the kinetic current densities for oxygen reduction on each catalyst measured at 0.90 $V_{\text {RHE. }}$ (d) Tafel plots for the catalysts. All of the NPs were loaded on carbon support. of these catalysts could be similar. From these results, we found that although Ir and Au catalysts do not show good activity for this reaction, the $\mathrm{Au}_{0.5} \mathrm{Ir}_{0.5}$ catalyst showed higher activity, which was comparable to that of Pt. This result is regarded as an example revealing the possibility of LDOS engineering as a rational strategy to design effective catalysts.

The same catalytic tests in $0.05 \mathrm{M} \mathrm{H}_{2} \mathrm{SO}_{4}$ aqueous solution were also performed (Fig. S14 $\dagger$ ). It was a little different from the alkaline case, ORR activity of $\mathrm{Au}_{0.5} \mathrm{Ir}_{0.5}$ was not comparable to that of Pt. However, the activity of Ir and Au was much worse than the alloy and the tendency was the same as the alkaline case. This might be caused by the nature that only Pt can exhibit high activity in both acidic and alkaline solutions, while Ir and Au show pretty low activity in acidic condition. Furthermore, we performed an accelerated durability test (ADT) to investigate the catalytic stability of $\mathrm{Au}_{0.5} \mathrm{Ir}_{0.5}$ catalyst by cyclic potential sweeps

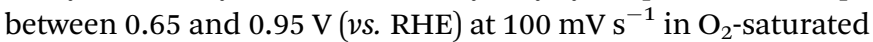
$1.0 \mathrm{M} \mathrm{NaOH}$ aqueous solution at room temperature. The integral of the hydrogen under potential deposition $\left(\mathrm{H}_{\mathrm{upd}}\right)$ region between 0.1 to $0.4 \mathrm{~V}$ ( $v s$. RHE) was compared (Table S13 $\dagger$ ). After 10000 cycles, the CV measurement showed a loss of only $7.9 \%$ in the $\mathrm{H}_{\text {upd }}$ peak and the catalyst still showed better activity for ORR than monometallic Ir or Au catalyst (Fig. S15†).

\section{Conclusions}

In summary, we successfully synthesized AuIr solid-solution alloy NPs over the entire composition range by a chemical reduction method, although $\mathrm{Au}$ and Ir are immiscible even in a high-temperature liquid phase in the bulk phase diagram. We demonstrated that Ir atoms on the $\mathrm{Au}_{0.5} \mathrm{Ir}_{0.5}$ alloy surface possess a Pt-like LDOS, and therefore, the alloy catalyst shows an excellent ORR activity comparable to that of the Pt catalyst, even though monometallic Ir or Au catalyst shows poor activity. This work represented untapped potentials of novel solidsolution alloy NPs of immiscible systems and proved the usefulness of LDOS engineering which is a rational strategy for designing and developing desirable catalysts.

\section{Conflicts of interest}

There are no conflicts to declare.

\section{Acknowledgements}

This research was supported by JST ACCEL programme Grant Number JPMJAC1501 and MEXT KAKENHI Grant-in-Aid for Young Scientists (B) 15K17831. STEM observations were performed as part of a programme conducted by the Advanced Characterization Nanotechnology Platform sponsored by the MEXT of the Japanese Government. Synchrotron XRD measurements were carried out at SPring-8 under proposal No. 2014B1382, 2015A1586 and 2016A1255. The activities of the INAMORI Frontier Research Centre, Kyushu University are supported by the KYOCERA Corporation. The authors thank M. Murase (Kyoto University) for assistance with the synthesis. 


\section{Notes and references}

1 C. Kittel, Introduction to Solid State Physics, John Wiely \& Sons, Inc, 8th edn, 2004.

2 R. Hoffmann, Solids and surfaces. A chemist's view of bonding in extended structures, Wiely-VCH, New York, 1988.

3 K. Kusada, H. Kobayashi, T. Yamamoto, S. Matsumura, N. Sumi, K. Sato, K. Nagaoka, Y. Kubota and H. Kitagawa, J. Am. Chem. Soc., 2013, 135, 5493-5496.

4 K. Kusada and H. Kitagawa, Adv. Mater., 2016, 28, 1129-1142.

5 X. Huang, S. Li, Y. Huang, S. Wu, X. Zhou, S. Li, C. L. Gan, F. Boey, C. A. Mirkin and H. Zhang, Nat. Commun., 2011, 2, 292.

6 F. Wang, K. Kusada, D. Wu, T. Yamamoto, T. Toriyama, S. Matsumura, Y. Nanba, M. Koyama and H. Kitagawa, Angew. Chem., Int. Ed., 2018, 0395, 4505-4509.

7 H. Kobayashi, K. Kusada and H. Kitagawa, Acc. Chem. Res., 2015, 48, 1551-1559.

8 K. Kusada, M. Yamauchi, H. Kobayashi, H. Kitagawa and Y. Kubota, J. Am. Chem. Soc., 2010, 132, 15896-15898.

9 K. Kusada, H. Kobayashi, R. Ikeda, Y. Kubota, M. Takata, S. Toh, T. Yamamoto, S. Matsumura, N. Sumi, K. Sato, K. Nagaoka and H. Kitagawa, J. Am. Chem. Soc., 2014, 136, 1864-1871.

10 E. R. Essinger-Hileman, D. DeCicco, J. F. Bondi and R. E. Schaak, J. Mater. Chem., 2011, 21, 11599.

11 Q. Zhang, K. Kusada, D. Wu, T. Yamamoto, T. Toriyama, S. Matsumura, S. Kawaguchi, Y. Kubota and H. Kitagawa, Nat. Commun., 2018, 9, 510.

12 H. Okamoto and T. B. Massalski, Bull. Alloy Phase Diagrams, 1984, 5, 381 .
13 Y. J. Song, Y. M. L. De Jesús, P. T. Fanson and C. T. Williams, Appl. Catal., B, 2014, 154-155, 62-72.

14 Y. Guan and E. J. M. Hensen, J. Catal., 2013, 305, 135-145.

15 A. Aguilar-Tapia, R. Zanella, C. Calers, C. Louis and L. Delannoy, Phys. Chem. Chem. Phys., 2015, 17, 2802228032.

16 X. Bokhimi, R. Zanella and C. Angeles-Chavez, J. Phys. Chem. C, 2010, 114, 14101-14109.

17 A. Gómez-Cortés, G. Díaz, R. Zanella, H. Ramírez, P. Santiago and J. M. Saniger, J. Phys. Chem. C, 2009, 113, 9710-9720.

18 H. a. Rojas, J. J. Martínez, G. Díaz and A. Gómez-Cortés, Appl. Catal., A, 2015, 503, 196-202.

19 Y.-J. Song, Y. M. López-De Jesús, P. T. Fanson and C. T. Williams, J. Phys. Chem. C, 2013, 117, 10999-11007.

20 S. H. Park, H. M. Park, S. S. Han, S. Y. Han and J. Y. Song, RSC Adv., 2016, 6, 3210-3212.

21 J. K. Nørskov, J. Rossmeisl, A. Logadottir, L. Lindqvist, J. R. Kitchin, T. Bligaard and H. Jónsson, J. Phys. Chem. B, 2004, 108, 17886-17892.

22 J. Zhang, M. B. Vukmirovic, Y. Xu, M. Mavrikakis and R. R. Adzic, Angew. Chem., Int. Ed., 2005, 44, 2132-2135.

23 S. Kawaguchi, M. Takemoto, K. Osaka, E. Nishibori, C. Moriyoshi, Y. Kubota, Y. Kuroiwa and K. Sugimoto, Rev. Sci. Instrum., 2017, 88, 085111.

24 a. R. Denton and N. W. Ashcroft, Phys. Rev. A: At., Mol., Opt. Phys., 1991, 43, 3161-3164.

25 F. H. B. Lima, J. Zhang, M. H. Shao, K. Sasaki, M. B. Vukmirovic, E. A. Ticianelli and R. R. Adzic, J. Phys. Chem. C, 2007, 111, 404-410.

26 P. P. Lopes, D. Strmcnik, J. S. Jirkovsky, J. G. Connell, V. Stamenkovic and N. Markovic, Catal. Today, 2015, 262, 41-47. 